\title{
Computational scaffold hopping: cornerstone for the future of drug design?
}

\author{
"...although computational scaffold hopping is an intellectually \\ stimulating scientific exercise, interests in scaffold hopping might \\ often be rather pragmatic in nature."
}

First draft submitted: 22 February 2017; Accepted for publication: 28 February 2017; Published online: 9 May 2017

Keywords: active compounds $\bullet$ computational methods $\bullet$ drug design $\bullet$ scaffold hopping

- therapeutic targets

\section{Scaffolds}

The 'scaffold' concept is widely applied in medicinal chemistry and drug design to generate, analyze, and compare core structures of active compounds and analog series [1]. Scaffolds might be defined algorithmically or on the basis of medicinal chemistry knowledge [1-3]. In computational analysis, a hierarchical definition of scaffolds has dominated the field for two decades [2]. On the basis of this definition, scaffolds are obtained from compounds by removal of substituents (R-groups) while retaining ring systems and linker fragments between rings [2]. It follows that each ring-containing compound yields a scaffold and that the addition of a ring to a compound (which may be regarded as an R-group) always generates a new scaffold. Recently, an alternative scaffold methodology has been introduced to further increase the medicinal chemistry relevance of computationally generated scaffolds [3]. This approach extracts scaffolds from analog series, rather than individual compounds, and takes reaction information into account [3]. Preferences for scaffold definitions may vary. However, for systematic computational analysis of scaffolds, the consistent application of a formal definition is essential [1].

\section{Scaffold hopping}

The 'scaffold hopping' concept originated from computational chemistry and virtual compound screening [4,5]. It refers to the search for compounds that have similar activity but contain different core structures. Besides activity, other molecular properties might also be considered. Thus, the goal of scaffold hopping is the identification of structurally diverse compounds that are similar in activity/property space [5]. Core structure modifications or replacements might be attempted on the basis of chemical knowledge, at least for individual compound series, but searching for scaffold hops requires computational frameworks. For benchmarking of virtual screening methods, the assessment of scaffold hopping potential has become a gold standard, which is not without problems. Scaffolds extracted from bioactive compounds represent a wide spectrum of structural relationships, ranging from chemically very similar scaffolds to others that are structurally unrelated. Benchmark studies reported in the literature are often statistical in nature and do not - or not sufficiently - specify how scaffold hopping is evaluated and whether or not different structural relationships have been considered [5]. While benchmarking is a necessary but not sufficient exercise for evaluating virtual screening methods, prospective scaffold hopping applications starting from known active compounds are more interesting. In medicinal chemistry and drug design, computational scaffold hopping aims at finding replacements of chemical liable compounds,

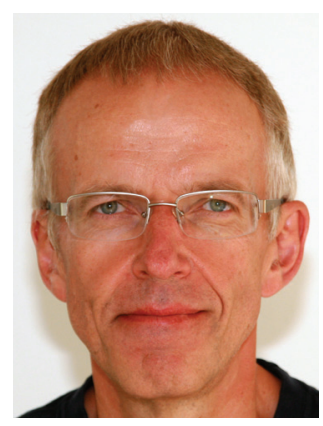

Jürgen Bajorath

Department of Life Science Informatics, B-IT, LIMES Program Unit Chemical Biology \& Medicinal Chemistry, Rheinische FriedrichWilhelms-Universität, Dahlmannstr. 2, D-53113 Bonn, Germany

Tel.: +492282699306

Fax: +492282699341

bajorath@bit.uni-bonn.de 
series with flat structure-activity relationships (SARs), or (natural) molecules that are difficult to access synthetically. In addition, the pharmaceutical industry, is also attempting to identify compounds that share activity characteristics with known candidates or drugs, but are chemically sufficiently different from them to help establish a competitive patent position. Thus, although computational scaffold hopping is an intellectually stimulating scientific exercise, interests in scaffold hopping might often be rather pragmatic in nature.

\section{"...if target proteins would not be capable of specifically interacting with structurally diverse compounds, scaffold hopping would not be feasible, regardless of the methods used."}

\section{Methods}

Regardless of methodological details, scaffold hopping relies on the assessment of molecular (dis) similarity, for which a variety of computational concepts have been developed [6]. Interestingly, computational scaffold hopping methods that evaluate molecular similarity in very different ways are successfully applied. From a principle point of view, computational methods based upon the pharmacophore concept should be preferred for scaffold hopping [4]. This is the case because pharmacophores, the 'business ends' of active compounds responsible for interactions with their targets, often only consist of R-groups (but not always). Accordingly, a given pharmacophore might be transferable from one scaffold to another, provided its geometric arrangement is retained. Therefore, pharmacophore searching of compound databases is a premier approach to scaffold hopping. Similarly, methods that search for molecules having similar shape taking chemical surface properties into account [7] should be well suited for scaffold hopping. However, many other similarity search and compound classification methods are also successfully applied. Among others, these include different similarity search and machine-learning approaches utilizing 2D molecular representations of varying complexity [5]. From a methodological viewpoint, it appears that for many compound classes and targets, 'anything goes'; for others, nothing. A possible rationalization of these observations is the ability of many - but not all - pharmaceutical targets to recognize structurally diverse compounds, as revealed by large-scale analyses of available active small molecules [8,9]. Clearly, if target proteins would not be capable of specifically interacting with structurally diverse compounds, scaffold hopping would not be feasible, regardless of the methods used. It follows that scaffold hopping potential of computational approaches cannot be separated from target characteristics and should hence be viewed in a broader context.

\section{New developments}

Recent trends in computational scaffold hopping include an increasing focus on natural products as starting points [10] and on methods for fragment replacements in active compounds [11-13]. Although scaffold hopping calculations were 'traditionally' carried out at the level of complete compounds, new search tools and descriptors have been introduced for comparing scaffolds directly and identifying promising replacements [13-15]. In these instances, scaffold similarity is assessed instead of whole molecule or pharmacophore similarity. Furthermore, biological profiles have also been used to establish activity relationships between compounds. For example, compound activity annotations from hundreds of assays have been encoded in fingerprint formats to quantify biological similarity and search for compounds having similar activity profiles [16]. Since no structural or chemical descriptors are used in this case, structurally distinct compounds can be identified. However, compounds with similar profiles in (phenotypic) cell-based or highcontent assays might act by different mechanisms or even on different targets. This requires careful consideration when evaluating the results of biologically driven scaffold hopping exercises. Combining compound and target descriptors in the search for new scaffolds is also possible, especially when applying machine-learning methods.

\section{The future}

What does the future hold? Although scaffold hopping plays a central role in medicinal chemistry and drug design, there are challenges ahead. Given the diversity of methods currently used in this field and their essentially unpredictable performance in practical applications, introducing new computational concepts with consistent scaffold hopping potential across different compound classes will have high priority. Retrospective benchmark calculations typically overestimate the ability of all scaffold hopping methods, be they simple or complex, to prospectively identify novel active compounds. Hence, more realistic application-oriented performance measures will also be required to guide method development. Furthermore, scaffold hopping potential should best be separately considered for 'easy' and 'hard' small-molecule targets, which has thus far only rarely been done, if at all. For easy targets, many computational methods might identify new active compounds often resembling others that are already available. The situation drastically changes when it comes to targets with only little available ligand information that are more resistant to small-molecule binding. Here, many scaffold hopping approaches fail, which leaves room for new developments that require less prior knowledge of active compounds.

Because computational scaffold hopping generally extrapolates from existing compounds, the ability to 
create chemical novelty is intrinsically limited, except if approaches are applied that exclusively use biological instead of structural descriptors. If biological similarity is a primary criterion for compound selection, experimental data must be collected in advance for scaffold hopping libraries, which limits the applicability of biological descriptors (in addition to potential mechanistic ambiguities). How to best abstract from chemical structure to create chemical novelty and how to balance important details and fuzziness of molecular representations are still open questions for which future research will hopefully provide answers.

At least one more critically important aspect should be mentioned. Scaffold hopping methods generally do not take the potency of template compounds into account and do not predict the potency level of candidates. In fact, the majority of successful scaffold hops yields weakly potent compounds for chemical exploration. To further increase the impact of computational scaffold hopping on drug design, the ability to prioritize potent candidate compounds would be an attractive goal. This might be achieved by applying scaffold hopping and potency prediction methods in concert or by developing new concepts that integrate scaffold hopping and potency prediction, which would be more exciting. Of course, aiming for higher-level predictions is not sufficient; reliability and consistency are essential to further advance the field.

\section{References}

1 Hu Y, Stumpfe D, Bajorath J. Computational exploration of molecular scaffolds in medicinal chemistry. J. Med. Chem. 59(9), 4062-4076 (2016).

2 Bemis GW, Murcko MA. The properties of known drugs. 1. Molecular frameworks. J. Med. Chem. 39(15), 2887-2893 (1996).

3 Dimova D, Stumpfe D, Hu Y, Bajorath J. ASB scaffolds: computational design and exploration of a new type of molecular scaffolds for medicinal chemistry. Future Sci. OA 2(4), FSO149 (2016).

4 Schneider G, Neidhart W, Giller T, Schmid G. 'Scaffoldhopping' by topological pharmacophore search: a contribution to virtual screening. Angew. Chem. Int. Ed. 38(19), 2894-2896 (1999).

$5 \mathrm{Hu}$ Y, Stumpfe D, Bajorath J. Recent advances in scaffold hopping. J. Med. Chem. 60(4), 1238-1246 (2017).

6 Maggiora G, Vogt M, Stumpfe D, Bajorath J. Molecular similarity in medicinal chemistry. J. Med. Chem. 57(8) 3186-3204 (2014).

7 Rush TS 3rd, Grant JA, Mosyak L, Nicholls A. A shape-based 3-D scaffold hopping method and its application to a bacterial protein-protein interaction. J. Med Chem. 48(5) 1489-1495 (2005).

8 Hu Y, Bajorath J. Global assessment of scaffold hopping potential for current pharmaceutical targets. Med. Chem. Commun. 1(5) 339-344 (2010).

\section{Conclusion}

Computational scaffold hopping is here to stay, no doubt, but to reach its full potential future improvements must go well beyond successful benchmark calculations. To these ends, realistically assessing method performance, better understanding fundamental and target-related constraints of scaffold hopping, and further increasing the success rate of practical applications are prime tasks. So far, neither computational scaffold hopping nor any other individual computational approach has put drug design up onto a new level. However, scaffold hopping belongs to the core of drug design methods and any substantially new developments in this field will experience a high level of interest, beyond the computational community.

\section{Financial \& competing interests disclosure}

The author has no relevant affiliations or financial involvement with any organization or entity with a financial interest in or financial conflict with the subject matter or materials discussed in the manuscript. This includes employment, consultancies, honoraria, stock ownership or options, expert testimony, grants or patents received or pending, or royalties.

No writing assistance was utilized in the production of this manuscript.

9 Hu Y, Bajorath J. Quantifying the tendency of therapeutic target proteins to bind promiscuous or selective compounds. PLoS ONE 10(5) e0126838 (2015).

10 Kawamura S, Unno Y, Hirokawa T, Asai A, Arisawa M, Shuto S. Rational hopping of a peptidic scaffold into non-peptidic scaffolds: structurally novel potent proteasome inhibitors derived from a natural product, belactosin A. Chem. Commun. 50(19) 2445-2447 (2014).

11 Vainio MJ, Kogej T, Raubacher F, Sadowski J. Scaffold hopping by fragment replacement. J. Chem. Inf. Model. 53(7) 1825-1835 (2013).

12 Saluste G, Albarran MI, Alvarez RM et al. Fragment-hoppingbased discovery of a novel chemical series of proto-oncogene PIM-1 kinase inhibitors. PLoS ONE 7(10) e45964 (2012).

13 Ertl P. Database of bioactive ring systems with calculated properties and its use in bioisosteric design and scaffold hopping. Bioorg. Med. Chem. 20 (18) 5436-5442 (2012).

14 Ertl P. Intuitive ordering of scaffolds and scaffold similarity searching using scaffold keys. J. Chem. Inf. Model. 54(6) 1617-1622 (2014).

15 Rabal O, Amr FI, Oyarzabal J. Novel scaffold fingerprint (SFP): applications in scaffold hopping and scaffold-based selection of diverse compounds. J. Chem. Inf. Model. 55(1) 1-18 (2015).

16 Petrone PM, Simms B, Nigsch F et al. Rethinking molecular similarity: comparing compounds on the basis of biological activity. ACS Chem. Biol. 7(8) 1399-1409 (2012). 